\title{
Compaction Behaviour of Alumina Powders Spray-Dried with Organic Binders
}

\author{
S. Baklouti $\left({ }^{1}\right)$, P. Coupelle $\left({ }^{2}\right)$, T. Chartier $\left({ }^{1, *}\right)$ and J.F. Baumard $\left({ }^{1}\right)$ \\ $\left({ }^{1}\right)$ LMCTS $\left({ }^{* *}\right)$, ENSCI, 47 avenue Albert Thomas, 87065 Limoges, France \\ $\left({ }^{2}\right)$ KPCL-Sapec, avenue du Président Kennedy, B.P. 806, 87015 Limoges, France
}

(Received 9 June 1996, accepted 12 July 1996)
PACS.81.20.Ev - Powder processing: powder metallurgy, compaction, sintering mechanical alloying, and granulation
PACS.81.20.Hy - Forming, molding, extrusion, etc.

\begin{abstract}
The compaction of spray-dried alumina, containing 3 wt. $\%$ organic binders (PVA or PEG), is analysed through the evolution of intergranular porosity. The variation of this porosity versus glass transition temperature of the binder and forming pressure is compared with the prediction of a model derived from a previous work on powder metallurgy.
\end{abstract}

Résumé. - La compaction de poudres d'alumine atomisées, contenant $3 \%$ en masse de liants organiques (PVA ou PEG), est analysée en suivant l'évolution de la porosité intergranulaire. La variation de cette porosité, en fonction de la température de transition vitreuse du liant et de la pression de mise en forme, est comparée à un modèle élaboré à partir de travaux réalisés sur la compaction de poudres métalliques.

\section{Introduction}

In ceramic processing it is often desirable to use powders with a mean grain size in the $\mu \mathrm{m}$ range, in order to get densification at relatively low temperatures and to control the microstructural evolution. The feed material used in a forming process such as uniaxial die pressing must satisfy some stringent requirements. First the powder should be free flowing in order to get reproducible behaviour of the powder bed in the mould before, during and after pressing. To meet this objective, powders are generally granulated by spray-drying with several added organic agents $[1,2]$. Among them, binders play an important role to increase the green strength of pressed products. The mean size of agglomerates obtained by spray-drying is typically $100-200 \mu \mathrm{m}$.

In earlier analyses of compaction of spray-dried powders, several steps have been described $[3,4]$. The first stage corresponds to rearrangement of granules. It is most often neglected since agglomerates are spherical and free flowing $[5,6]$. The second stage involves elimination of the intergranular porosity through plastic deformation and/or fracturing of granules, and the third stage deals with the rearrangement of elementary ceramic particles within the former granules

(*) Author for correspondence

$\left({ }^{* *}\right)$ URA CNRS 320

(C) Les Éditions de Physique 1996 
and a decrease of the intragranular porosity. Of course, these stages overlap to a certain extent, although they occur more or less consecutively [6].

Recently several works aimed at investigating the deformation behaviour of single porcelain [7] and single alumina [8] agglomerates prepared by spray-drying. It was shown that, during compaction, spray-dried agglomerates exhibit plastic behaviour in a significant range of deformation before crushing. Then the model developed by Arzt and Helle $[9,10]$, on the compaction of monosized, plastic particles in powder metallurgy, could be adapted to the case of spray-dried ceramic materials, during the second stage at moderate pressure compaction.

According to several earlier works on the compaction of metallic powders and by using a simple law of granular deformation, the purpose of the present paper is to model the compaction behaviour of spray-dried alumina.

\section{Experimental Procedure}

Aqueous suspensions of an alumina ceramic powder with a mean grain size of $1.3 \mu \mathrm{m}$ (Pechiney P152SB, Gardanne, France) were spray-dried with organic binders in a pilot plant equipment. The binders used were a partially hydrolysed polyvinyl alcohol PVA ( $\mathrm{M}_{\mathrm{W}}=31000$, Hoechst 4-88, D) and a modified polyethylene glycol PEG $\left(\mathrm{M}_{\mathrm{W}}=20000\right.$, Union Carbide, PEG20M, USA). According to literature data, the glass transition temperatures $T_{\mathrm{g}}$ of the dry polymers are $\approx 352 \mathrm{~K}$ for PVA and $\approx 213 \mathrm{~K}$ for PEG [11], if one assimilates the glass transition temperatures of $\mathrm{PEG}$ and polyethylene oxide. It is common to use such water soluble polymers to dry-press ceramic powders; their respective $T_{\mathrm{g}}$ are respectively beyond and below room temperature, at which experiments described below took place.

Aqueous suspensions for spray-drying were prepared with an alumina concentration of 60 wt. $\%$ solids. The mean size of the spray-dried agglomerates was about $200 \mu \mathrm{m}$. The total organic content of granulated powders was $3 \mathrm{wt} \%$ with respect to alumina. The temperature and the flow of the incoming drying air were adjusted to get a residual moisture content of $0.6 \mathrm{wt} . \%$ with respect to alumina. Powders spray-dried with PEG and PVA will be denoted by symbols 1 and 2, respectively. A sample of powder 2 (containing PVA) was also subsequently dried at $100{ }^{\circ} \mathrm{C}$ for 2 hours in an oven to get moisture-free agglomerates (and therefore moisture-free PVA binder). Such a powder will be denoted by symbol 3. To investigate the compaction behaviour, powders were loaded into a cylindrical lubricated steel die and compacted at a rate of $4 \mathrm{~mm} \mathrm{~min}^{-1}$ in an universal mechanical testing machine. The green samples were then analysed by standard $\mathrm{Hg}$ porosimetry.

\section{Results and Discussion}

The type of the binder significantly affected the microstructure of the green bodies. For instance, the inspection of the surface of samples pressed at $80 \mathrm{MPa}$ reveals that the intergranular porosity has been fully eliminated for powder 1, for which PEG actually constitutes a ductile binder (Fig. 1a). For powder 3, where the binder is now dry PVA, the intergranular porosity is still present (Fig. 1c). The differing abilities of the granules to deform can explain the observed changes in the microstructure.

As mentioned earlier, the glass transition temperature of $\mathrm{PEG}$ is much below room temperature, and that of dry PVA is much higher. As the binder content was 3 wt.\%, the moisture content of $0.6 \mathrm{wt} . \%$ corresponds to more than $20 \mathrm{wt} . \%$ water content in the binder if moisture is only absorbed in the organic phase. According to several authors [3], the $T_{\mathrm{g}}$ of this waterplasticized PVA should be located in the vicinity of room temperature. This is the reason why the microstructure of the green bodies pressed with powder 2 indicates an intermediate aspect 


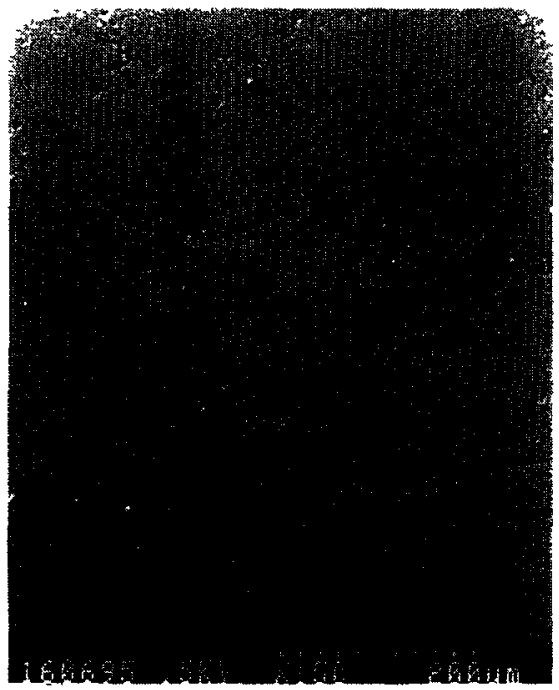

a)

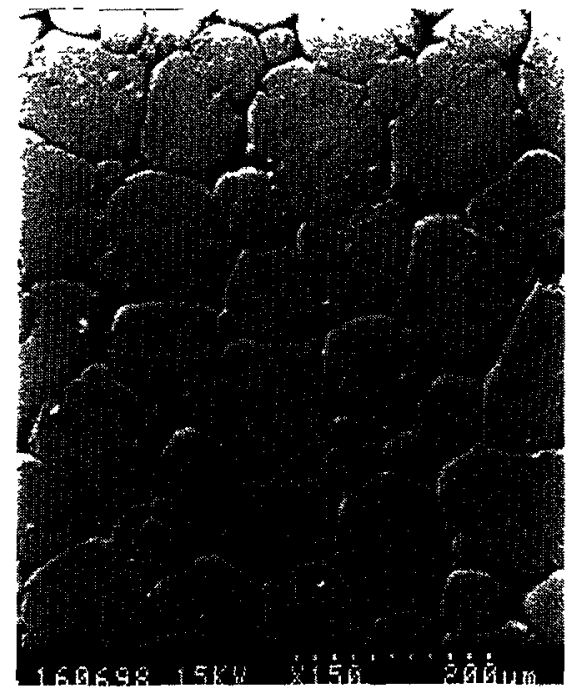

b)

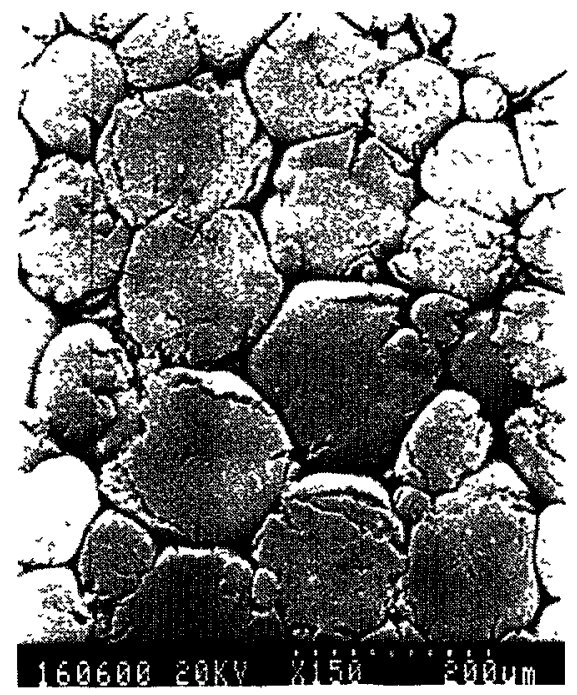

c)

Fig. 1. - SEM micrographs of the surface of samples prepared from: a) powder 1, b) powder 2 and c) powder 3 , pressed at $80 \mathrm{MPa}$.

between those of powders 1 and 3 (Fig. 1b). The ability of a powder to forming by uniaxial pressing is generally characterized by the variation of relative density versus applied pressure. As emphasized in a previous work [12], the evolution of the density with pressure results from two phenomena: the elimination of intergranular porosity at low/moderate pressure, followed by the elimination of the intragranular porosity at larger pressure.

The porosity of the green samples pressed with the three powders was investigated in order to estimate the respective contributions of the decrease of the intergranular porosity and of the decrease of the intragranular porosity on the densification. Figure 2 shows an example of 


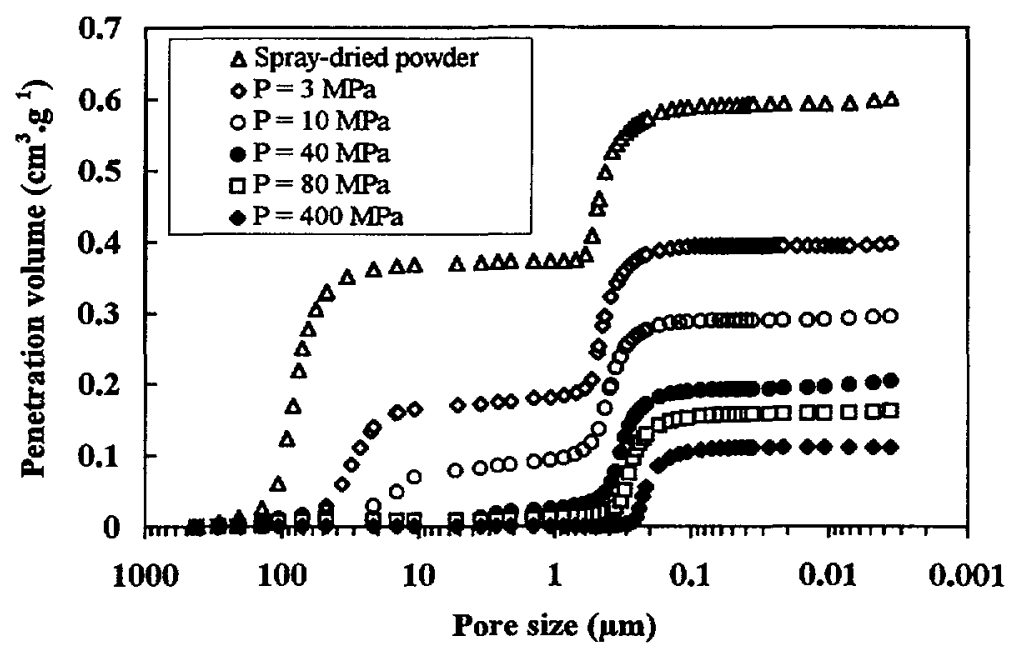

Fig. 2. - Mercury penetration of alumina spray-dried powders (with 3 wt.\% PVA - powder 2) compacted at different pressures.

data obtained on samples pressed with powder 2. It is obvious that the $\mathrm{Hg}$ penetration takes place in two steps for the spray-dried powder. According to the results depicted in Figure 2, the mean equivalent pore size in the granules lies in the range $0.3-0.5 \mu \mathrm{m}$, a value which is not unexpected when one considers the mean grain size of the alumina used as the starting material $(1.3 \mu \mathrm{m})$. The pore size at which $\mathrm{Hg}$ penetration takes place into the granules is lower than $1 \mu \mathrm{m}$, and as a consequence, we consider that $\mathrm{Hg}$ penetration into pore sizes larger than $1 \mu \mathrm{m}$ could be attributed to intergranular porosity. As the compaction pressure increases, this porosity decreases almost down to zero, then intragranular porosity begins to decrease during the third stage as described previously [6].

The variations of intergranular and intragranular porosities are plotted versus forming pressure in Figure 3. These curves suggest that the differences in the compaction behaviour of the three powders are mainly related to the variation of the intergranular porosity itself. One can notice that while this porosity decreases rapidly just after compaction at low pressures, the variations become smoother as the density of the sample increases, and that large forming pressures are required to eliminate coarse interstices between spray-dried agglomerates. Also, in agreement with results published previously in the literature [4], the densification is enhanced when the glass transition temperature $T_{\mathrm{g}}$ of the binder shifts to lower temperatures, especially below the ambient temperature. This can be undoubtedly attributed to the enhanced deformability of granules. However, the effect is less pronounced during the third stage since the intragranular porosity seems less sensitive to the binder system. The most important step for densification seems to be the second stage, during which the granules deform against each other under the compressive load. The morphological evolution of the granule assembly presents high similarities with metallic powders, if one excepts the limited decrease of the intragranular porosity which does not occur for dense metallic particles [13].

The model which we use below is based on the work of Arzt, Fischmeister and Helle [9,10,14], who investigated the behaviour of metallic powders during cold or hot isostatic pressing, the densification of these materials being due to plastic deformation. The model in question links the variation of the compactness $\gamma$ for an assembly of monosized and spherical particles to the 


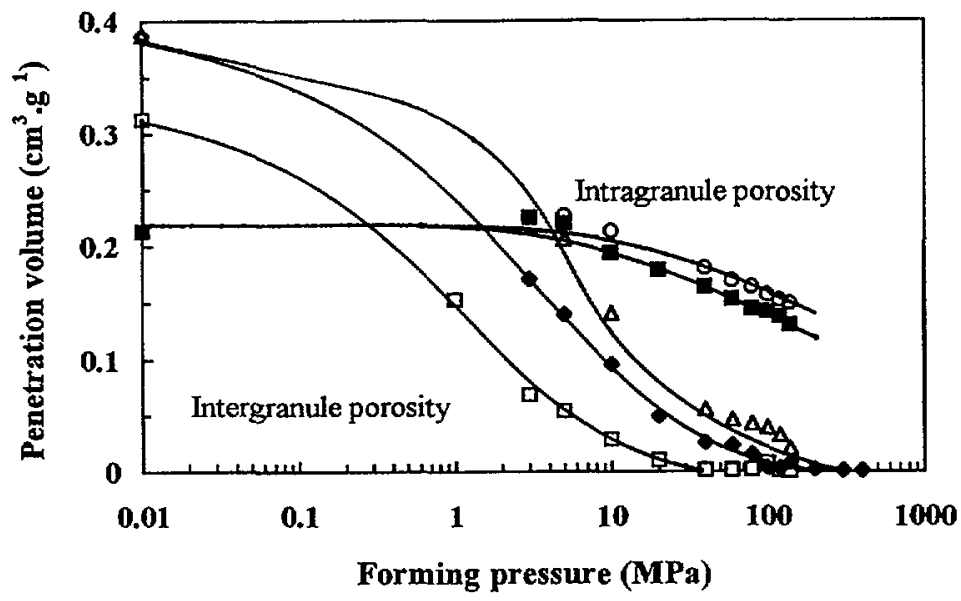

Fig. 3. - Variation of intergranular porosity: $(\square)$ powder $1,(\bullet)$ powder 2, $(\Delta)$ powder 3 and of intragranular porosity $(O)$ powder $2,(\mathbb{0})$ powder 3 with forming pressure.

applied pressure $P$. Compaction is described as a fictitious increase of the particle diameter, the whole volume of the sample being kept constant. Once the spheres have grown to a new radius, some of them overlap and the excess material is transported from the contact zone to the free surfaces within the porosity. A salient feature of the model is the variation of the mean coordination number of particles during the process.

For a pressure $P$ applied to the sample, the force $F$ acting on a boundary between two particles is given by [15]:

$$
\frac{F}{A}=\frac{\pi D^{2} P}{A(\gamma) Z(\gamma) \gamma}
$$

where $A$ is the surface of the boundary, $Z$ the coordination number and $D$ the diameter of particles. A further assumption concerns the relation between the contact surface $A$ and the force $F$ applied to this contact through [7]:

$$
F=k A
$$

$k$ being a parameter related to the "hardness" of the granule. This simple equation is derived from the Hertzian analysis of contact mechanics, and has been used by several authors in their investigation of the behaviour of agglomerates submitted to uniaxial compression [7].

A further step requires additional knowledge on the variation of parameters $Z$ and $A$ with respect to compactness $\gamma$. The original expressions given by Arzt were later simplified by Helle [10], who proposed convenient approximations:

$$
\begin{aligned}
& A=\frac{\pi\left(\gamma-\gamma_{0}\right) D^{2}}{12\left(1-\gamma_{0}\right)} \\
& Z=12 \gamma
\end{aligned}
$$

where $\gamma_{0}$ is the compactness of the feed powder in the die before pressure is applied. Insertion of equations (3) and (4) into equation (1) leads to:

$$
\frac{P}{k}=\frac{\left(\gamma-\gamma_{0}\right) \gamma^{2}}{\left(1-\gamma_{0}\right)}
$$




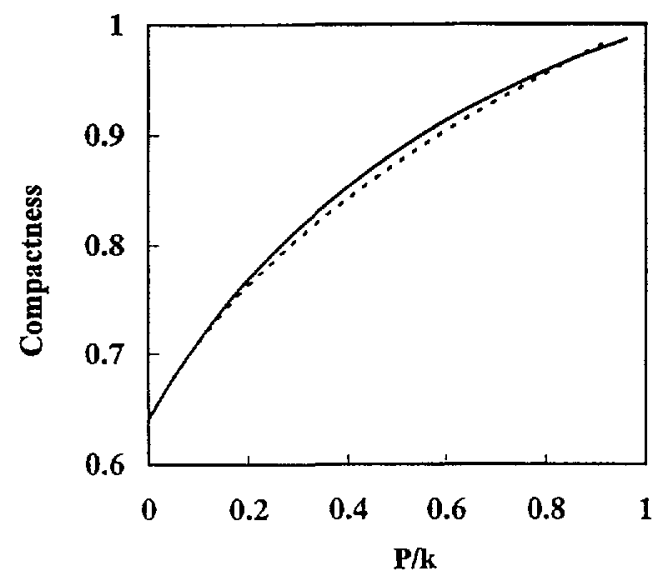

Fig. 4. - Variation of compactness versus normalized coordinate $P / k,(-)$ according to the full calculations derived from the work of Arzt [9]. (.. ) according to the simplified equation (5).

Thus pressure $P$ and compactness $\gamma$ are simply interrelated through $\gamma_{0}$ and $k$. It is worthwhile to mention that according to equation (5), the compaction behaviour mainly depends on $k$, since increasing values of $k$ are expected to move the data towards larger pressures, and at $P=k$ the intergranular porosity is virtually eliminated (e.g. $\gamma=1$ ). A plot of $\gamma$ versus normalized coordinate $P / k$ is depicted in Figure 4, where the results can be compared with those obtained from the original expressions for $Z$ and $A$ proposed by Arzt [9]. A good agreement is observed. Let us mention finally that the calculations have been made here with the assumption of a random dense packing of the powder before compaction. The value of $\gamma_{0}$ used in this calculation $\left(\gamma_{0}=0.64\right)$ is then somewhat larger than the value commonly accepted in ceramic literature for loose powders $\left(\gamma_{0}=0.5-0.55\right)[16]$.

The intergranular porosity $E$ expressed in vol. \% is now given by:

$$
E=1-\gamma
$$

In Figure 5 the variation of the intergranular porosity (vol.\%), as well as the variation predicted by equation (5), are plotted versus the forming pressure for powders 1,2 and 3 . Although such a model predicts a rapid decrease of the intergranular porosity, its applicability proves to be limited, especially for samples formed in the high pressure range. For the three powders studied, intergranular porosity persists at large pressure and decrease asymptotically, once the residual porosity is smaller than about $13 \%$. This phenomenon indicates an increasing resistance of granules to deformation. This resistance is larger when the $T_{\mathrm{g}}$ of the binder increases as mentioned earlier and revealed in Figure 1.

This simple model is based on the equation $F=k A$. This relation actually comes up against two difficulties. First, the growth of the contact area $A$ between granules which, at a certain stage, brings contacts close enough on a given agglomerate surface to form triple lines, at which three agglomerates come in contact. The formation of these triple lines impedes further growth of such contacts. Fishmeister has suggested [14] to call this last stage of compaction the stage of "constraint hardening". An estimation of the compactness at which triple lines start to form is straightforward from the model and the obtained value $(\gamma=0.87)$ actually coincides with the compactness at which the experimental data deviate from the prediction made from the model. 


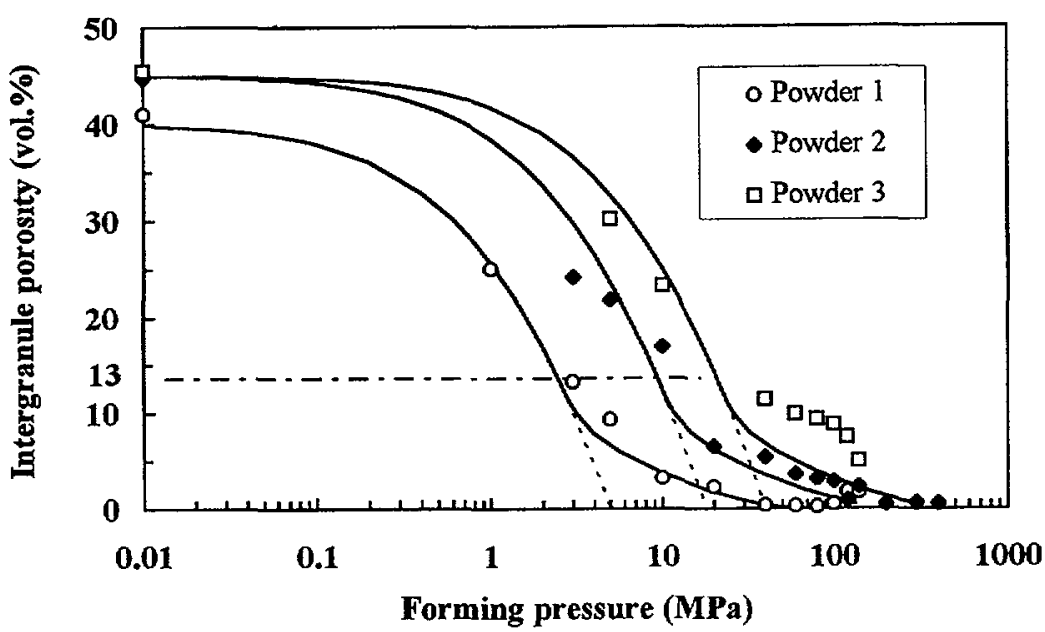

Fig. 5. - Variation of intergranular porosity versus forming pressure for the three investigated powders. $(\cdots)$ variation predicted by equation (5). (-) variation predicted by equation (10). $k=50,200$ and $400 \mathrm{MPa}$ for powders 1,2 and 3 respectively.

The second difficulty is related to the use of equation (2) itself. During compaction, the granules themselves densify since the intragranular porosity starts to decrease before the full elimination of intergranular porosity (Fig. 3). The intragranular porosity, which is attributed to particle rearrangements within the granules, decreases at pressures beyond $10 \mathrm{MPa}$ for the three powders investigated. Consequently, for powders containing PVA (powder 2 and mostly powder 3), the intragranular porosity starts to decrease whereas the intergranular porosity has not fully disappeared, i.e. when the granules must still deform. In this situation, the hardness of the granules is expected to increase and to make more difficult the elimination of the last fraction of the intergranular porosity.

It is a difficult task, at this moment, to introduce contributions of these hardening phenomena in the previous model. However, Fischmeister [14] suggested an approach to include the influence of the first one. During the final stage of compaction, samples can be considered as diphasic and containing zones where the material is "hard" owing the presence of triple lines and regions where the deformation is still unconstrained. The volume fraction of these regions changes conversely during compaction. The resistance to deformation may be expressed as a linear combination of the properties of the components, so that:

$$
P=f_{\mathrm{h}} P_{\mathrm{h}}+f_{\mathrm{s}} P_{\mathrm{s}}
$$

where $f_{\mathrm{h}}$ and $f_{\mathrm{s}}$ denote the volume fraction of the "harder" and "softer" components, and $P_{\mathrm{h}}$ and $P_{\mathrm{s}}$ mean the yield criteria for constrained and unconstrained deformation respectively. The expression of $P_{\mathrm{s}}$ is given by equation (5) whereas the expression of $P_{\mathrm{h}}$ can be borrowed from the theory of extrusion and adapted to the present geometry:

$$
P_{\mathrm{h}}=\frac{2 k}{3} f_{\mathrm{h}} \operatorname{Ln}\left[\frac{A(1) Z(1)}{A(1) Z(1)-A(\gamma) Z(\gamma)}\right]
$$

$f_{\mathrm{h}}(\gamma)$ is approximately given by a cubic equation [14]:

$$
f_{\mathrm{h}}(\gamma)=1-f_{\mathrm{s}}(\gamma)=455(\gamma-0.87)^{3} \quad \text { with } \quad 0.87 \leq \gamma \leq 1
$$


We note that $f_{\mathrm{h}}(0.87)=0$ and $f_{\mathrm{h}}(1)=1$ as stated before.

Insertion of equations (5) and (8) in equation (7) leads finally to:

$$
\frac{P}{k}=\left[\frac{\gamma^{2}\left(\gamma-\gamma_{0}\right)}{1-\gamma_{0}}\left(1-f_{\mathrm{h}}\right)+\frac{2}{3} f_{\mathrm{h}} \operatorname{Ln} \frac{1-\gamma_{0}}{\left(1-\gamma_{0}\right)-\left(\gamma-\gamma_{0}\right) \gamma}\right]
$$

Again, as in equation (5), the only adjustable parameters are the granule hardness $k$ and the initial compactness $\gamma_{0}$.

The variation of the intergranular porosity could be reproduced satisfactorily in the case of the powder spray-dried with PEG, while significant deflections from the predicted behaviour are observed for powders spray-dried with PVA, mostly when the organic binder is moisturefree. The "constraint hardening" suggested by Fishmeister [14] is not sufficient to explain the total hardening of granules. This behaviour may probably be attributed to the reduction of the intragranular porosity, whereas the intergranular porosity has not fully disappeared, and then to the increase in the hardness of the granules themselves.

\section{Conclusion}

The main conclusions of the present work are listed below:

- the resistance of granules to deformation depends strongly on the nature of the binder and particularly on its glass transition temperature,

- the variation of the intergranular porosity observed for the three spray-dried powders emphasizes the role of the organic binder on the granule deformability,

- the model developed from the work of Arzt and Helle on compaction of metallic powders seems adapted to describe the variation of the intergranular porosity versus forming pressure in the case of spray-dried powders, if one assumes a gradual increase of the agglomerate hardness during pressing and particularly when the compactness of the pressed sample is larger than $87 \%$,

- finally a good densification is obtained if the intergranular porosity is totally eliminated before a reduction in the intragranular porosity itself.

\section{References}

[1] Lahnstein R. B., Organische Additive für Trockenpressverfahren, Keramische Zeitschrift 42 (1990) 156.

[2] Busch T., Schweizer D. and Sorg C., Spray Granulation of Alumina with Organic Binders, cfi/Ber. DKG 68 (1991) 527.

[3] Reed J. S., in "Introduction to the Principles of Ceramic Processing" (John Wiley \& Sons, New York, 1988) pp. 327-354.

[4] Nies C. W. and Messing G. L., Effect of Glass-Transition Temperature of Polyethylene Glycol-Plasticized Polyvinyl Alcohol on Granule Compaction, J. Am. Ceram. Soc. 67 (1984) 301. 
[5] Heckel R. W., An Analysis of Powder Compaction Phenomena, Trans. Met. Soc. AIME 221 (1961) 1001.

[6] Van Der Zwan J., The compaction and Mechanical Properties of Agglomerated Material, C. A. M. Siskens, Powder Technology 33 (1982) 43.

[7] Coupelle P., Destermes J., Miglioretti F. et Baumard J.F., Déformation des granules d'atomisation sous compression uniaxiale, L'Industrie Céramique nº61 (1991) 408.

[8] Agniel Y., Rôle des propriétés des granules pour la fabrication de pièces de poudres céramiques granulées sans défauts de compaction, $\mathrm{PhD}$ Thesis (Lyon, France, 1992).

[9] Arzt E., The influence of an Increasing Particle Coordination on the Densification of Spherical Powder, Acta Met. 30 (1982) 1883.

[10] Helle A.S., Easterling K.E. and Ashby M.F., Hot-Isostatic Pressing Diagrams: New Developments, Acta Met. 33 (1985) 2163.

[11] van Krevelen D. W., In "Properties of Polymers", $3^{\text {rd }}$ Edition (Elsevier, Amsterdam, 1990).

[12] Baklouti S., Pressage de poudres d'alumine atomisées : Influence des liants organiques sur l'aptitude au pressage, PhD Thesis (Limoges, France, 1996).

[13] Youshaw R.A. and Halloran J.W., Compaction of Spray-dried Powders, Am. Ceram. Soc. Bull. 61 (1982) 227.

[14] Fishmeister H.F. and Arzt E., Densification of Powders by Particle Deformation, Powder Metallurgy 26 (1983) 82.

[15] Molerus O., Theory of Yield of Cohesive Powders, Powder Technology 12 (1975) 259.

[16] Kendall K., Behaviour of Particle Assemblies-Relevance to Ceramic Processing, Materials Forum 11 (1988) 61. 